\title{
The effect of Benson relaxation technique on fatigue of patients diagnosed with multiple sclerosis
}

\author{
Abeer Hisham Alzaghmouri', Rami Masa'Deh²*, Mira Al Jaberi³, Omayma M. Masadeh', Ahmad M Al Smadi5, \\ Ghadeer Ahmad Alshawabkeh ${ }^{6}$
}

'Department of Clinical Nursing, Applied Science Private University, Amman, Jordan, ${ }^{2}$ Department of Psychiatric and Community Health Nursing, School of Nursing, Applied Science Private University, Amman, Jordan, ${ }^{3}$ MD student, School of Medicine, University of Jordan, Amman, Jordan, ${ }^{4}$ Psychological and Educational Counseling, Relief International, Amman, Jordan, ${ }^{5}$ Department of Neurology, Jordan University Hospital, Amman, Jordan, ${ }^{6}$ Department of Research and Education, Research Triangle Institute, Amman, Jordan

\begin{abstract}
Introduction: The previous studies showed that fatigue is a very common symptom in patients diagnosed with multiple sclerosis (MS) and has the greatest effect on their activities of daily living. Benson relaxation technique (BRT) is one of the most relaxation techniques used to decrease fatigue, improve emotional status and quality of life in patients with several chronic diseases. This study aimed to investigate the effect of BRT on fatigue level of patients diagnosed with MS in Jordan.

Methods: This is a quasi-experimental repeated measure study. It involved six neurological clinics in the Ministry of Health and five neurological clinics at private hospitals. In this study, 95 patients returned the completed questionnaire and attended BRT.

Results: The mean age of the patients was 33 years. Females accounted for $60 \%$ of the patients and almost half of the patients were single. All patients reported having a university degree and three-quarters of the patients were employed. Almost, two-thirds of the patients reported having not more than 1 time of relapse in the past 2 years. The majority of them reported $<3$ years from the confirmed diagnosis. Findings showed a statistical significant reduction in the overall level of fatigue including physical, cognitive, and psychosocial fatigue of patients with MS after BRT $(p<0.001)$.

Conclusions: Results suggested that this technique is a very effective strategy for reducing fatigue in patients with MS. Therefore, in addition to prescribing medication, it is recommended that this method be applied in the care of patients diagnosed with MS.
\end{abstract}

Keywords: Multiple sclerosis; Benson; relaxation; fatigue; Jordan

\section{INTRODUCTION}

Multiple sclerosis (MS) is a chronic autoimmune, inflammatory neurological disease that affects the central nervous system of the individual, with an unknown etiology (1). According to Atlas of MS, the estimated number of individuals diagnosed with MS worldwide increased to 2.8 million in $2020(2,3)$. The prevalence of MS is increasing globally, with approximately $36 / 100,000$ population; most cases are among young people, and women have twice the risk of men (3-5). Prevalence is expected to continue rising in the Middle East, in line with worldwide trends (6).

Symptoms experienced by individuals diagnosed with MS vary depending on the location and size of involved areas of the central nervous system (7). Some symptoms

*Corresponding author: Rami Masa'Deh, Applied Science Private University, Amman, Jordan. E-mail: r_masadeh@asu.edu.jo; Phone: +962 796067657

Submitted: 30 March 2021/Accepted: 01 October 2021

DOI: https://doi.org/10.17532/jhsci.2021.1229 commonly include pain, sensory problems, visual disturbances, elimination dysfunction, impaired mobility, mood changes, cognitive changes, and fatigue $(8,9)$. Studies have shown that the very common symptom of fatigue - defined as an individual perception of deficiency in the physical or mental energy which affect the daily activities (10) - is particularly significant in its impacts on the activities of daily living of individuals diagnosed with MS $(11,12)$. Fatigue plays a major role in the MS disease process. It is usually present at the time of diagnosis and in most cases represents a main complaint of individuals seeking neurological consultation (13). Unfortunately, fatigue remains one of the most difficult symptoms to manage $(14,15)$.

Fatigue affects the overall quality of life, daily life activities, overall well-being, social relationships, and employment status of individuals diagnosed with MS, as well as imposing financial burdens on them, their families, and their communities $(16,17)$. The concatenation of diverse factors associated with fatigue for individuals diagnosed with MS underscores the need for research to develop optimum early 
interventions/management for fatigue among such service users.

The previous studies highlighted the importance of multidisciplinary care, including both advanced pharmacological and non-pharmacological approaches, in the management of fatigue in individuals diagnosed with MS $(1,18)$. Several non-pharmacological techniques have been used to manage fatigue (19), including physical rehabilitation and physical exercise/fitness, which were found to have positive impacts on level of fatigue and quality of life of individuals diagnosed with MS (20). Moreover, behavioral therapy (including energy management education and self-management) and relaxation techniques (such as reflexology and massage therapy) were found to be effective (21-23).

Benson relaxation technique (BRT) is one of the most popular relaxation techniques used to decrease fatigue, and improve emotional status, sleeping, and quality of life in individuals with several chronic diseases $(24,25)$. BRT has been found to be effective in managing fatigue in individuals with chronic renal failure undergoing hemodialysis, individuals with heart failure, and those diagnosed with MS (24-26). However, while BRT seems to be effective in managing fatigue in individuals with chronic diseases, its impact on fatigue among people diagnosed with MS in Jordan has not been examined. Furthermore, most previous studies focused on the severity of fatigue as an outcome variable. Therefore, this study aimed to investigate the effect of BRT on multidimensional fatigue levels of individuals diagnosed with MS to answer the following research question: Is there an effect of BRT on reducing the fatigue level of individuals diagnosed with MS?

\section{METHODS}

Study is designed as repeated measure pre-post quasi-experimental study. The study involved six neurological clinics in the Ministry of Health and five neurological clinics at private hospitals. All of them are located in Amman. The study population included all individuals diagnosed with MS in Jordan. A non-random convenience sampling technique was used to recruit individuals. The inclusion criteria were: (a) Having a medical diagnosis of MS and (b) being able to understand, read, and write Arabic. Exclusion criteria were: (a) Having a psychotic disorder (as documented in their medical file), (b) receiving psychotherapy during the previous 6 months, (c) attendance of any intervention targeting physical health and/or fatigue during the previous 6 months, and (d) absence from more than 2 of the BRT intervention sessions. Data were collected from June 2020 to December 2020. The sample size was calculated using G power software (27). Taking into consideration, the main statistical tests used in this study (i.e., paired $t$-test), assuming a power of $80 \%$, an $\alpha$ level of 0.05 , and medium effect size, a total of 92 individuals were deemed sufficient to detect any effect of BRT on patient fatigue. In this study, 130 individuals were approached and 122 consented to participate; of these, 95 completed and returned the questionnaire, representing a $78 \%$ response rate. As study instruments, we used:

- Sociodemographic characteristics: Age, gender, level of education, employment status, marital status, duration of disease, and frequency of relapse during the preceding 2 years.

- Arabic version of Modified Fatigue Impact Scale (FIS): The FIS was initially developed in 1994 (28), which was superseded by the Modified FIS (MFIS). MFIS has been examined with 180 individuals diagnosed with MS from different European countries (29) and translated into 30 languages, including Arabic $(30,31)$. It displays good validity and reliability, with Cronbach's alpha coefficient values ranging from 0.77 to 0.90 in many previous studies and different languages, including Arabic (30-34). The Arabic version of MFIS was used in this study, and participants were asked about the effect of fatigue during the past 4 weeks. The assessment included 21 items with a scale of $0-4$ points $(0=$ never, $1=$ rarely, $2=$ sometimes, $3=$ often, and 4 = almost always). Items on the MFIS can be aggregated into three subscales, with a range of scores: Physical subscale (ranging from 0 to 36), cognitive subscale (ranging from 0 to 40), and psychosocial subscale (ranging from 0 to 8 ), as well as into a total MFIS score (ranging from 0 to 84 ). All items are scaled so that higher scores indicate a more significant impact of fatigue on individuals' activities. In this study, the Arabic version of MFIS showed good reliability, with a Cronbach's alpha of 0.87 for the total score ranging from 0.85 and 0.90 for all subscales.

- $\quad$ BRT: The interventionist was one of the research team members who are a licensed expert in relaxation techniques, holding a professorship in psychosocial health. Individuals received two sessions of BRT, each of $1 \mathrm{~h}$ duration, in a private room provided by the selected hospitals/clinics. Individuals performed BRT in the presence of the interventionist, who provided feedback by evaluating the participants' skills in performing the technique, and ensured that had acquired sufficient skills to do it alone. A video of BRT was displayed to participants during the sessions. Moreover, a CD of BRT was provided, with handout copies about the performance of BRT, so individuals could watch the video and read the instructions when needed. Individuals were instructed to perform the BRT according to the following steps:

- Sit quietly in a comfortable position.

- Close your eyes.

- Deeply relax all your muscles, beginning at your feet, progressing up to your face, and keep them deeply relaxed.

- Breath through your nose. Become aware of your breathing. As you breathe out, say the word "one" silently to yourself. Breathe in deeply for $3 \mathrm{~s}$ through the nose; hold the breath for $3 \mathrm{~s}$; then exhale slowly for four seconds through the mouth.

- Continue for $10 \mathrm{~min}$. You may open your eyes to check the time, but do not use an alarm.

- When you finish, sit quietly for several minutes at first with closed eyes, and later with opened eyes.

- Do not worry about whether you are successful in achieving a deep level of relaxation. Maintain a passive attitude and permit relaxation to occur at its own pace.

- When distracting thoughts occur, ignore them, and continue repeating "one." With practice, the response 
should come with little effort. Practice the technique twice a day, but not within $2 \mathrm{~h}$ after any meal, as the digestive processes seem to interfere with the elicitation of anticipated changes.

Individuals were instructed to perform the BRT 2 times a day for $10 \mathrm{~min}$ at home, for 8 weeks, at two specific times, with $7-8 \mathrm{~h}$ intervals between each episode. The participants were emphasized not to forget to perform the technique. However, if they forget to do it, they were asked to do it as soon as possible.

There was a self-reporting "performance record form" which was filled out daily by the participants, to ensure their BRT compliance. Every week, the interventionist met the participants to encourage them to perform the technique and to collect their "performance record form." Moreover, to ensure that the participants were performing the intervention appropriately, they performed the technique again in the presence of the interventionist.

The interventionist established a group on WhatsApp for all the participants and sent them the BRT video twice a day at specific times, to remind the participants to perform the technique. Furthermore, an identification card of the interventionist was given to the participants, and they were asked to contact the interventionist if they had any questions regarding the technique.

Before starting with the data collection process, ethical approvals were gained from the Institutional Review Board of the Applied Science Private University and from the Jordanian Ministry of Health to collect data from governmental hospitals (IRB number: 2020-2021-1-1). These were presented to the private sector hospitals/clinics involved in the study, who subsequently granted access. Participants' rights, such as voluntary participation, privacy, confidentiality, and the right to withdraw at any time without giving a reason, were guaranteed and explained to all participating individuals. Participants were informed that all collected data was securely stored (i.e., in locked filing cabinets and password-protected computers), accessible only to the research team, and that no personally identifying information would be reported in this study.

An invitation letter and an information sheet were sent to potential participants. The researchers' contact details were provided on the information sheet for those who wanted to participate or who had any enquiries about the study. An informed consent form was signed by those who agreed to participate in the study. Those who wanted to participate and were willing to attend the program were included in the study. The participants then filled out questionnaires before the implementation of the program. The course was then implemented over 8 weeks. After that, participants filled out the questionnaires again.

Data were analyzed using the SPSS version 25 (35). All numbers in the results were rounded up to the closest two decimal points. The level of significance was set as 0.05 , therefore, any $p<0.05$ was considered significant. Descriptive statistics were used to describe the sociodemographic characteristics. A paired $t$-test was conducted to check any differences in fatigue before and after implementing the course. An independent $t$-test and a one-way analysis of variance (ANOVA) were conducted to examine

the difference in fatigue and its subscales according to gender, education, employment status, time since diagnosis, and number of relapse events.

\section{RESULTS}

Table 1 presents the sociodemographic characteristics of the individuals diagnosed with MS. Individuals reported an approximate mean age of 33 years. Exactly $60 \%$ of participants were female, and almost half of them were single. All participants reported having a university degree (the majority of them had baccalaureate degrees), and three-quarters were employed. Almost two-thirds reported having not more than 1 time of relapse in the preceding 2 years. Individuals were asked about the time elapsed since diagnosis, the majority of them reported that they had been diagnosed for $<3$ years.

Paired samples $t$-test was conducted to investigate the BRT impact on the level of overall fatigue, including physical, cognitive, and psychosocial subscales in individuals diagnosed with MS (Table 2). First of all, the difference in level of overall fatigue pre- and post-intervention was checked. Findings showed that there were significant reductions in the levels of overall fatigue after attending BRT (post-intervention). The mean level of fatigue decreased from $48.82 \pm$ 14.95 pre-intervention to $34.34 \pm 11.59$ post-intervention ( $\mathrm{t}[94]=13.79, p<0.001)$. The magnitude of the difference was checked, revealing eta-squared $=0.6$, indicating a large effect size.

With regard to the fatigue subscales, the mean level of physical subscale was significantly decreased from $21.62 \pm 6.28$ pre-intervention to $13.39 \pm 4.64$ post-intervention $(\mathrm{t}[94]=17.92$, $p<0.001)$. The magnitude of the difference was checked; eta-squared $=0.7$ indicates a large effect size. Moreover, the

TABLE 1. Sociodemographic characteristics of participants ( $N=95)$

\begin{tabular}{lc}
\hline Variable & $\mathrm{N}(\%)$ or M \pm SD \\
\hline Age (years) & $32.85 \pm 5.70$ \\
Gender & \\
Male & $38(40)$ \\
Female & $57(60)$ \\
Marital status & \\
Single & $49(51.6)$ \\
Married & $43(45.3)$ \\
Divorced & $3(3.1)$ \\
Education & \\
Baccalaureate & $82(86.3)$ \\
Master & $13(13.7)$ \\
Employment status & \\
Employed & $72(75.8)$ \\
Unemployed & $23(24.2)$ \\
Duration of disease (years) & \\
$<1$ & $10(10.5)$ \\
$1-2$ & $39(41.1)$ \\
$2-3$ & $33(34.7)$ \\
$>3$ & $13(13.7)$ \\
Relapses during the past 2 years & \\
0 & $33(31.4)$ \\
1 & $34(32.4)$ \\
2 & $14(13.3)$ \\
3 & $24(22.9)$ \\
\hline
\end{tabular}


TABLE 2. Pre- and post-intervention fatigue levels ( $\mathrm{N}=95)$

\begin{tabular}{lcccr}
\hline Variable & $\begin{array}{c}\text { Pre-intervention } \\
\mathrm{M} \pm \mathrm{SD}\end{array}$ & $\begin{array}{c}\text { Post-intervention } \\
\mathrm{M} \pm \mathrm{SD}\end{array}$ & $\mathrm{t}$ & $p$-value \\
\hline Overall fatigue & $48.82 \pm 14.95$ & $34.34 \pm 11.59$ & 13.79 & $<0.001$ \\
Physical fatigue & $21.62 \pm 6.28$ & $13.39 \pm 4.64$ & 17.92 & $<0.001$ \\
Cognitive fatigue & $17.74 \pm 6.76$ & $13.42 \pm 5.65$ & 9.19 & $<0.001$ \\
Psychosocial fatigue & $4.71 \pm 1.99$ & $3.21 \pm 1.77$ & 5.61 & $<0.001$ \\
\hline
\end{tabular}

mean level of cognitive subscale was significantly decreased from $17.74 \pm 6.76$ pre-intervention to $13.42 \pm 5.65$ post-intervention ( $\mathrm{t}[94]=9.19, p<0.001)$. The magnitude of the difference was checked; eta-squared $=0.4$ indicates a large effect size. Finally, the mean level of psychosocial subscale was significantly decreased from $4.71 \pm 1.99$ pre-intervention to $3.21 \pm 1.77$ post-intervention $(\mathrm{t}[94]=5.61, p<0.001)$. The magnitude of the difference was checked; eta-squared $=0.2$ indicates a large effect size.

An independent samples $t$-test was conducted to investigate the difference in fatigue and its subscales according to gender, education, and employment status before the intervention. Results showed no significant differences in fatigue according to any of the above-mentioned variables. Moreover, a one-way ANOVA to examine the difference in fatigue and its subscales according to time since diagnosis and number of relapses showed no significant differences in the above-mentioned variables.

\section{DISCUSSION}

Fatigue is a common and pervasive MS symptom, which impairs activities of daily living and quality of life among individuals diagnosed with MS. This study aimed to examine the effect of BRT on MS patient fatigue. The mean age of individuals diagnosed with MS in Jordan, according to the random sample used in this study, was 33 years, with females representing $60 \%$ of the individuals; these characteristics reflect global trends, as the average age of MS diagnosis worldwide is around 32 years old, and females are almost twice as likely to live with MS than males (3). In the Middle East and North Africa, the mean age of individuals diagnosed with MS is around 29 years, and the female-tomale ratio ranges from 1.3:1 in some countries to $1.8: 1$ in others (5).

The findings of this study showed that the majority of the individuals had baccalaureate degrees, and three-quarters of them were employed. This level of education is expected, as all individuals were young, and data from the Jordanian Department of Statistics showed that the percentage of people having a bachelor degree and above for those older than 22 years is $95 \%$ of the population, and the unemployment rate for the year 2020 was $24 \%$ (36). The results of this study showed that almost two-thirds of the individuals reported having one relapse incident during the preceding 2 years. Similarly, the previous international studies form the United States and United Kingdom showed that individuals diagnosed with MS had approximately one to two relapses over a 2-year period $(37,38)$.

This study delivered BRT to individuals diagnosed with MS, and findings showed a significant reduction in the overall level of fatigue after the intervention. Fatigue among patients with MS was found to be improved after muscle relaxation technique (39). In the same line, BRT was applied in a clinical trial study conducted in Iran among 60 individuals diagnosed with MS divided into two equal experimental and control groups showed that the mean level of fatigue was significantly reduced after the intervention for those who attended BRT sessions (26). One explanation of this reduction could be due to BRT turning off the fightor-flight response, and reducing the level of neurotransmitters, which brought the body back to a pre-stress state. This, in turn, reduced muscle tension and minimized negative impacts, such as fatigue (40). Consistently, another study compared the effects of reflexology and relaxation on pain in women suffering from MS and showed a significant decrease in pain 3 months following the intervention (41). It seems that relaxation methods could decrease fatigue and improve quality of life in individuals with chronic diseases, particularly MS.

The Arabic MFIS used in this study had three subscales (i.e., physical, cognitive, and psychosocial). The results showed that the mean levels of physical, cognitive, and psychosocial fatigue subscales were significantly decreased post-intervention. This means that the effect of fatigue on overall physical activity, cognitive and emotional status, and socializing with others was reduced after the intervention. This corroborates some previous studies which reported that relaxation techniques improved physical fatigue (24). This is because relaxation of the muscles releases hormones and other chemicals in the body, causing physical changes, like increased blood flow in the hands and feet, and reduced severity of fatigue (24). Second, similar to findings of the current study, cognitive fatigue was found to be reduced in a randomized clinical trial of 60 individuals after attending BRT (42). One explanation of this reduction is that cognitive fatigue in MS is related to parasympathetic activity, reduced responsiveness, and an inflammatory process in the brain (43). BRT could rebalance the neurotransmitters, parasympathetic activity, and therefore improve cognitive fatigue. Finally, a systematic review of 20 studies concerning fatigue in individuals diagnosed with MS concluded that psychological interventions were found to decrease MS-related psychosocial fatigue in all reviewed studies (44). It seems that there is consensus in the literature that relaxation interventions could improve physical, cognitive, and psychosocial fatigue in individuals diagnosed with MS.

\section{CONCLUSIONS}

BRT is based on mind control and muscle relaxation. The results of this study showed that this technique is a very effective strategy for reducing fatigue in Individuals diagnosed with MS. Therefore, it is recommended that this method be applied in the care of these individuals as a complementary therapy alongside conventional pharmacological treatment for fatigue. To improve the early management 
of fatigue related to MS, individuals should be transferred to specialized centers that apply relaxation techniques. Health authorities should facilitate this transition. Relaxation should decrease their fatigue and improve their quality of life. Moreover, the findings of this study form the baseline for future studies examining the effect of relaxation techniques on fatigue in individuals diagnosed with MS in the region. Future research could examine the effectiveness of other non-pharmacological interventions on fatigue among individuals diagnosed with MS, and compare their results with those achieved using BRT.

Although this study is the first to apply BRT in Jordan and examine its effect on fatigue among individuals diagnosed with MS, the study did not contain a control group. Therefore, a randomized clinical trial in future research may be needed. Moreover, most of the participants in this study were diagnosed with MS during the preceding 3 years, and patients with longer history may have different experiences in response to BRT. Furthermore, it would be enlightening to include older patient populations, who may have more disabilities causing additional problems with fatigue.

\section{COMPETING INTEREST}

The authors declare that they have no conflicts of interest.

\section{ACKNOWLEDGMENT}

The authors are grateful to the Applied Science Private University, Amman, Jordan, for the partial financial support granted to this research project.

\section{REFERENCES}

1. Correale J, Gaitán MI, Ysrraelit MC, Fiol MP. Progressive multiple sclerosis: From pathogenic mechanisms to treatment. Brain 2017;140(3):527-46. https://doi.org/10.1093/brain/aww258.

2. Browne $P$, Chandraratna $D$, Angood C, Tremlett $H$, Baker $C$, Taylor BV, et al. Atlas of multiple sclerosis 2013: A growing global problem with widespread inequity. Neurology 2014;83(11):1022-4. https://doi.org/10.1212/wnl.0000000000000768.

3. Walton C, King R, Rechtman L, Kaye W, Leray E, Marrie RA, et al. Rising prevalence of multiple sclerosis worldwide: Insights from the Atlas of MS, third edition. Mult Scler 2020;26(14):1816-21. https://doi.org/10.1177/1352458520970841.

4. Schriefer D, Ness NH, Haase R, Ziemssen T. Gender disparities in health resource utilization in patients with relapsing-remitting multiple sclerosis: A prospective longitudinal real-world study with more than 2000 patients. Therapeutic advances in Neurological Disorders 2020;1-13

https://doi.org/10.1177/1756286420960274.

5. Heydarpour P, Khoshkish S, Abtahi S, Moradi-Lakeh M, Sahraian MA. Multiple sclerosis epidemiology in middle East and North Africa: A systematic review and meta-analysis. Neuroepidemiology 2015;44(4):232-44.

https://doi.org/10.1159/000431042.

6. Yamout BI, Assaad W, Tamim H, Mrabet S, Goueider R. Epidemiology and phenotypes of multiple sclerosis in the Middle East North Africa (MENA) region. Mult Scler J Exp TransI Clin 2020;6(1):1-6.

https://doi.org/10.1177/2055217319841881.

7. Bishop M, Rumrill PD. Multiple sclerosis: Etiology, symptoms, incidence and prevalence, and implications for community living and employment. Work 2015;52(4):725-34. https://doi.org/10.3233/wor-152200.

8. Chaudhary A, Chaudhary B. Common Invisible Symptoms of Multiple Sclerosis; 2020.

9. Disanto G, Zecca C, MacLachlan S, Sacco R, Handunnetthi L, Meier UC et al. Prodromal symptoms of multiple sclerosis in primary care. Ann Neurol 2018;83(6):1162-73

https://doi.org/10.1002/ana.25247.

10. Heine M, van den Akker LE, Blikman L, Hoekstra T, van Munster E, Verschuren $O$ et al. Real-time assessment of fatigue in patients with multiple sclerosis: How does it relate to commonly used self-report fatigue questionnaires? Arch Phys Med Rehabil 2016;97(11):1887-94.e1.

https://doi.org/10.1016/j.apmr.2016.04.019.

11. Yusuf $F$, Wijnands JM, Kingwell E, Zhu F, Evans C, Fisk JD, et al. Fatigue, sleep disorders, anaemia and pain in the multiple sclerosis prodrome. Mult Scler 2021;27(2):290-302.

https://doi.org/10.1177/1352458520908163.

12. de Heredia-Torres MP, Huertas-Hoyas E, Sánchez-Camarero C Máximo-Bocanegra N, Alegre-Ayala J, Sánchez-Herrera-Baeza P, et al. Occupational performance in multiple sclerosis and its relationship with quality of life and fatigue. Eur J Phys Rehabil Med 2020;56(2):148-54.

https://doi.org/10.23736/S1973-9087.20.05914-6.

13. Sellitto G, Morelli A, Bassano S, Conte A, Baione V, Galeoto G, et al. Outcome measures for physical fatigue in individuals with multiple sclerosis: A systematic review. Expert Rev Pharmacoecon Outcomes Res 2021;21(4):625-46.

https://doi.org/10.1080/14737167.2021.1883430

14. Chen MH, Wylie GR, Sandroff BM, Dacosta-Aguayo R, DeLuca J, Genova HM. Neural mechanisms underlying state mental fatigue in multiple sclerosis: A pilot study. J Neurol 2020;267(8):2372-82. https://doi.org/10.1007/s00415-020-09853-w.

15. Lindermann S. Fatigue Management in Occupational Therapy for People with Multiple Sclerosis: A Scoping Review; 2020.

16. van Zanten JV, Douglas MR, Ntoumanis N. Fatigue and fluctuations in physical and psychological wellbeing in people with multiple sclerosis: A longitudinal study. Mult Scler Relat Disord 2021;47:102602. https://doi.org/10.1016/j.msard.2020.102602.

17. Eizaguirre MB, Ciufia N, Roman MS, Martínez Canyazo C, Alonso R, Silva B, et al. Perceived fatigue in multiple sclerosis: The importance of highlighting its impact on quality of life, social network and cognition. Clin Neurol Neurosurg 2020;199:106265. https://doi.org/10.1016/j.clineuro.2020.106265.

18. Capone F, Motolese F, Falato E, Rossi M, Di Lazzaro V. The potential role of neurophysiology in the management of multiple sclerosis-related fatigue. Front Neurol 2020;11:251.

https://doi.org/10.3389/fneur.2020.00251.

19. Moss-Morris R, Harrison AM, Safari R, Norton S, van der Linden ML, Picariello F, et al. Which behavioural and exercise interventions targeting fatigue show the most promise in multiple sclerosis? A systematic review with narrative synthesis and meta-analysis. Behav Res Ther 2021;137:103464.

https://doi.org/10.1016/j.brat.2019.103464.

20. Any DA, Sanda J, Zorina SA, Silviu DA, Daniel DA. The role of physical exercise in multiple sclerosis. Ovidius Univ Ann Ser Phys Educ Sport Sci Mov Health 2020;20(1):10-5.

21. Patt N, Kool J, Hersche R, Oberste M, Walzik D, Joisten N, et al. High-intensity interval training and energy management education, compared with moderate continuous training and progressive muscle relaxation, for improving health-related quality of life in persons with multiple sclerosis: Study protocol of a randomized controlled superiority trial with six months' follow-up. BMC Neurol 2021;21(1):1-10.

https://doi.org/10.1186/s12883-021-02084-0.

22. Ebrahimi N, Loripour M, Sayadi A, Ardakani AM, Sayadi AR. Effect of foot reflexology on fatigue, stress and serum cortisol levels in women with multiple sclerosis. J Evol Med Dent Sci 2020;9(35):2497-502.

https://doi.org/10.14260/jemds/2020/543.

23. Frost-Hunt A. Effects of massage therapy on multiple sclerosis: A case report. Int Ther Massage Bodywork 2020;13(4):35. https://doi.org/10.3822/ijtmb.v13i4.545.

24. Seifi L, Ghezeljeh TN, Haghani H. Comparison of the effects of Benson muscle relaxation and nature sounds on the fatigue in patients with heart failure: A randomized controlled clinical trial. Holist Nurs Pract 2018;32(1):27-34. https://doi.org/10.1097/hnp.0000000000000242.

25. Elsayed EB, Radwan EH, Elashri NI, El-Gilany AH. The effect of Benson's relaxation technique on anxiety, depression and sleep quality of elderly patients undergoing hemodialysis. Int J Nursing Didactics 2019;9(2):23-31. https://doi.org/10.15520/ijnd.v9i02.2443.

26. Mirhosseini S, Mohammadi A, Rezaei M, Ajorpaz NM. The effect of benson relaxation technique on the fatigue severity of patients with MS. Journal of Client-Centered Nursing Care 2019;5(3):1.

https://doi.org/10.32598/jccnc.5.3.175.

27. Faul F, Erdfelder E, Buchner A, Lang AG. Statistical power analyses using G*Power 3.1: Tests for correlation and regression analyses. Behav Res Methods 2009;41(4):1149-60.

https://doi.org/10.3758/brm.41.4.1149

28. Fisk JD, Ritvo PG, Ross L, Haase DA, Marrie TJ, Schlech WF. Measuring the functional impact of fatigue: Initial validation of the fatigue impact scale. Clin Infect Dis 1994;18 Suppl 1:S79-83.

https://doi.org/10.1093/clinids/18.supplement_1.s79.

29. Kos D, Kerckhofs E, Carrea I, Verza R, Ramos M, Jansa J.Evaluation of the modified 
fatigue impact scale in four different European countries. Mult Scler 2005;11(1):76-80. https://doi.org/10.1191/1352458505ms1117oa.

30. Alawami AS, Abdulla FA. Psychometric properties of an Arabic translation of the modified fatigue impact scale in patients with multiple sclerosis. Disabil Rehabil 2020;28:1-9.

https://doi.org/10.1080/09638288.2020.1731853.

31. Khalil H, Al-Shorman A, Alghwiri AA, Abdo N, El-Salem K, Shalabi S, et al. Cross cultural adaptation and psychometric evaluation of an Arabic version of the modified fatigue impact scale in people with multiple sclerosis. Mult Scler Relat Disord 2019;39:101878.

https://doi.org/10.1016/j.msard.2019.101878.

32. Kos D, Kerckhofs E, Nagels G, D'Hooghe BD, Duquet W, Duportail M, et al. Assessing fatigue in multiple sclerosis: Dutch modified fatigue impact scale. Acta Neurol Belg 2003;103(4):185-91.

https://doi.org/10.1186/1471-2377-6-27.

33. Ghajarzadeh M, Jalilian R, Eskandari G, Ali Sahraian M, Reza Azimi A. Validity and reliability of persian version of modified fatigue impact scale (MFIS) questionnaire in Iranian patients with multiple sclerosis. Disabil Rehabil 2013;35(18):1509-12. https://doi.org/10.3109/09638288.2012.742575.

34. Larson RD. Psychometric properties of the modified fatigue impact scale. Int J MS Care 2013;15(1):15-20 https://doi.org/10.7224/1537-2073.2012-019.

35. IBM Corp., IBM SPSS Statistics for Windows; 2017.

36. Department of Statistics. Jordanian Statistics; 2020. Available from: http://www. dosweb.dos.gov.jo [Last accessed on 2021 Mar 22].

37. Nazareth TA, Rava AR, Polyakov JL, Banfe EN, Waltrip li RW, Zerkowski KB, et al. Relapse prevalence, symptoms, and health care engagement: Patient insights from the multiple sclerosis in America 2017 survey. Mult Scler Relat Disord 2018;26:219-34. https://doi.org/10.1016/j.msard.2018.09.002.

38. Multiple Sclerosis Trust. Replase in Multiple Sclerosis; 2020. Available from: https://www.mstrust.org.uk/about-ms/ms-symptoms-and-relapses/managing-relapses\#: : text=a\%20uk\%20study $\% 20$ in $\% 202012$,years $\% 20$ without $\% 20$ having $\% 20 a \% 20$ relapse [Last accessed on 2021 Mar 22].

39. Kamkar MZ, Maghsoudlou A. The effect of progressive muscle relaxation technique on the quality of sleep and fatigue in patients with multiple sclerosis. Nursing Midwifery J 2018;15(12):911-20.

40. Jourabchi Z, Satari E, Mafi M, Ranjkesh F. Effects of Benson's relaxation technique on occupational stress in midwives. Nursing 2020;50(9):64-8. https://doi.org/10.1097/01.nurse.0000694836.00028.28.

41. Nazari F, Soheili M, Hosseini S, Shaygannejad V. A comparison of the effects of reflexology and relaxation on pain in women with multiple sclerosis. J Complement Integr Med 2016;13(1):65-71.

https://doi.org/10.1515/jcim-2015-0046.

42. Akbari A, Ahmadi F, Jalili E, Khazaei S. The effect of relaxation technique (Jacobsen and Benson) on depression, anxiety, and stress in patients with multiple sclerosis. Curr Psychiat Res Rev 2020;16(3):213-9. https://doi.org/10.2174/2666082216999200819105928.

43. Sander $C$, Modes F, Schlake HP, Eling P, Hildebrandt H. Capturing fatigue parameters: The impact of vagal processing in multiple sclerosis related cognitive fatigue. Mult Scler Relat Disord 2019;32:13-8.

https://doi.org/10.1016/j.msard.2019.04.013

44. Phyo AZZ, Demaneuf T, de Livera AM, Jelinek GA, Brown CR, Marck CH, et al. The efficacy of psychological interventions for managing fatigue in people with multiple sclerosis: A systematic review and meta-analysis. Front Neurol 2018;9:149. https://doi.org/10.3389/fneur.2018.00149. 